\title{
Electrospun Nanofibre Membrane Based Transparent Slippery Liquid-Infused Porous Surfaces with Icephobic Properties
}

Advanced Materials Research Group, Faculty of Engineering, The University of Nottingham, Nottingham, NG7 2RD, UK

\section{Corresponding Author: Dr. Xianghui Hou}

\section{E-mail: xianghui.hou@nottingham.ac.uk Tel: +44-1159513920}

\section{Abstract}

Icephobic surfaces have attracted increasing attention due to their wide ranging application areas from wind and solar energy systems to aviation. Slippery liquid-infused porous surfaces (SLIPS) are being explored for passive ice protection due to their lower ice adhesion strength. In this study, we present a cost-effective and scalable electrospinning technique to produce freestanding nanofibrous polymeric surfaces for the fabrication of transparent icephobic SLIPS. The diameter of the electrospun fibres produced varied from 200 to $400 \mathrm{~nm}$ and the membranes had a theoretical porosity of $71.6 \pm 4.1 \%$. Furthermore, three different lubricants polychlorotrifluoroethylene oil (PcTFE), silicone oil and liquid paraffin, were used and it was observed that when silicone oil and PcTFE were used as lubricants for SLIPS, they provided high optical transparency (>90\%) in the visible light spectrum compared to PVDF-co-HFP itself. All SLIPS were subjected to centrifugal ice adhesion testing which revealed their ice adhesion strengths lower than $1 \mathrm{KPa}$ with significant delay in droplet icing compared to aluminium reference, from 5 up to $41 \mathrm{sec}$. The results indicated that enhanced icephobic properties of electrospun membranes have been clearly demonstrated.

Keywords: Slippery liquid-infused porous surfaces (SLIPS), electrospun, nanofibres, icephobic surface, ice adhesion strength. 


\section{Introduction}

Ice accretion often causes serious problems in many areas such as decreased efficiency of energy systems (i.e. wind turbines, solar panels), delays for air transportation or personal injuries from falling ice masses and structural damage of buildings due to the excessive weights of ice [1-4]. Many approaches to prevent surfaces from ice-causing problems have been investigated such as active heating systems [5], chemical de-icing fluids [6] (typically composed of ethylene glycol or propylene glycol) and mechanical removal [7]. These approaches either have considerable energy consumption or bring no negligible environmental impacts. As such, producing surfaces with anti-icing or icephobic properties to reduce the impact of the ice accretion is of vital importance to many industrial services.

Aizenberg et al. [8] proposed slippery liquid-infused porous surfaces (SLIPS) inspired from the Nepenthes pitcher plant. This structure consists of two main parts: 1) porous surface and 2) lubricant, and had remarkable slippery behaviour against immiscible liquids. Although there are several challenges remaining for the application of SLIPS in harsh conditions such as evaporation of lubricants, durability of the structure or the contamination of the surface with dust, significant icephobic properties have been achieved. Subramanyam et al. [9] focused on the effect of the texture density of silicon microposts on the ice adhesion properties of the SLIPS. They produced micropost surfaces using photolithography techniques and chose silicon oil and tetramethyl tetraphenyl trisiloxane as the lubricants. The results showed that the increasing texture density of the surface led to decreased ice adhesion strength. Dou et al. [10] reported a study showing a polyurethane anti-icing coating with an aqueous lubricating layer and ice adhesion strength as low as $30 \mathrm{KPa}$ was obtained. Erbil et al. [11] produced SLIPS with hydrophobic polypropylene sorbent mats and hydrophilic cellulose-based filter paper surfaces as porous structure, with different hydrophobic and hydrophilic liquids as lubricants. It was suggested that the hydrophilic lubricant impregnated hydrophilic porous surface would 
be promising candidates for anti-icing application because of their improved droplet icing times. Wang et al. [12] produced SLIPS by infusing perfluorinated lubricant into smooth/ hierarchical structured surfaces and found that low surface energy is a critical issue for the sliding speed. Zhu et al. [13] prepared SLIPS using rough polydimethylsiloxane (PDMS) coatings by adding silica nanoparticles. Silicon oil was infused to produce SLIPS and $75 \mathrm{KPa}$ of ice adhesion strength obtained. Chen et al. [14] produced four different structures (hydrophobic, superhydrophobic, silicic and fluorous slippery coatings) and evaluated their performance in anti-icing applications. They demonstrated that SLIPS differed from other surfaces in frost growth mechanism and frost formation time which provides better anti acing and de-icing properties.

For icephobic coatings on solar energy harvesting systems, windows and curtain walls, transparency has critical importance [15]. If the layer is not sufficiently optical transparent, some of the sunlight would be difficult to reach the solar systems which significantly affects the efficiency of the solar cells. However, there are only a limited number of study about transparent icephobic coatings [15-18]. Wu et al. produced transparent icephobic coatings using bio-based epoxy and reported a $50 \mathrm{KPa}$ ice adhesion strength was obtained at $-20{ }^{\circ} \mathrm{C}$ with a transmittance as high as $81 \%$ [15]. Chen et al. produced self-cleaning icephobic surfaces using modified $\mathrm{SiO}_{2}$ nanoparticles and an approximate $58 \mathrm{KPa}$ ice adhesion strength was obtained at $-15^{\circ} \mathrm{C}$ with the transmittance of $97.8 \%$ [16]. In another study, Chen et al. produced porous cellulose lauroyl ester films using nanoprecipitation technique into which perfluoropolyether was infused into the pores to obtain SLIPS. It was reported that this SLIPS had good anti-icing properties with the transmittance between 30 and $80 \%$ [17]. To understand the ice nucleation process, Shen et al. [19] investigated the effects of nanostructural features on interfacial ice nucleation and it was found that during the freezing process, the solid-liquid contact type determined the macroscopic freezing process. 
75 Up to now, different production methods were employed for the production of porous surfaces 76 for icephobic SLIPS, such as self-assembly [20, 21], laser writing [22, 23], phase separation

77 [24] and electrospraying [25]. These methods have their own advantages as well as 78 disadvantages such as necessity to high laser energies [26], technical barriers for large-scale 79 implementations [27], and limitation in polymer selection [28]. In this study, we present a 80 cost-effective and scalable electrospinning technique to produce freestanding nanofibrous 81 polymeric surfaces for the fabrication of transparent icephobic SLIPS. Three types of lubricants

82 (silicone oil, fluorinated oil and paraffin liquid) were used for the preparation of the SLIPS and 83 the key properties including droplet icing time, ice adhesion strength and optical transmittance 84 in the visible light spectrum were investigated.

\section{MATERIALS and METHODS}

\subsection{Materials and substrates}

87 For the production of electrospun membrane, Poly (vinylidene fluoride-cohexafluoropropylene) (PVDF-co-HFP) with average $\mathrm{M}_{\mathrm{W}}$ 400.000, average $\mathrm{M}_{\mathrm{n}}$ 130.000, dimethylformamide (DMF, >99\%) and acetone (>99.9\%) was purchased from Sigma Aldrich (UK). Fluorinated lubricant Poly (chlorotrifluoroethylene) (PcTFE) was kindly supplied by Halocarbon (USA). Other two types of lubricants (silicon oil and paraffin wax oil) were supplied from Aldrich. The chemical structures of the materials are shown in Figure 1. For the icephobic comparison purpose, AL2024-T3 aluminum alloy was used as reference. All chemicals were used as received, without further purification. 
a)

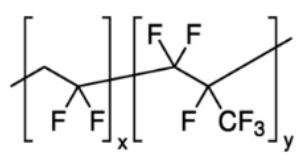

c)

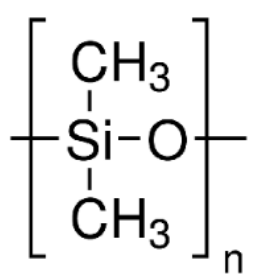

b)

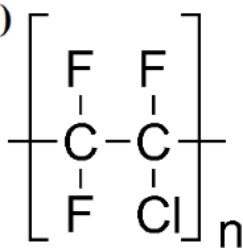

d)

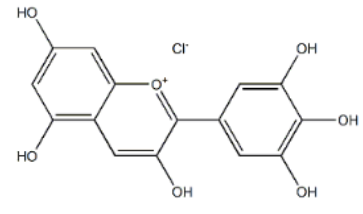

Figure 1. Chemical structures of (a) PVDF-co-HFP, (b) PcTFE (c) silicone oil and (d) paraffin liquid

\subsection{Methods}

100 For the process of electrospinning, 15 wt. \% concentration of PVDF-co-HFP solution was 101 prepared using DMF and acetone mixture (50:50 wt. \%). The applied voltage was chosen as $10217.5 \mathrm{kV}$ with $1.25 \mathrm{ml} / \mathrm{h}$ flow rate and $13 \mathrm{~cm}$ collector-needle tip distance. The thickness of the 103 electrospun membrane was controlled by the deposition time of fibres, and approximately 40 $104 \mu \mathrm{m}$ thickness was obtained with 3 hour deposition time. The schematic diagram of the electrospinning process is given in Figure 2 (a). 

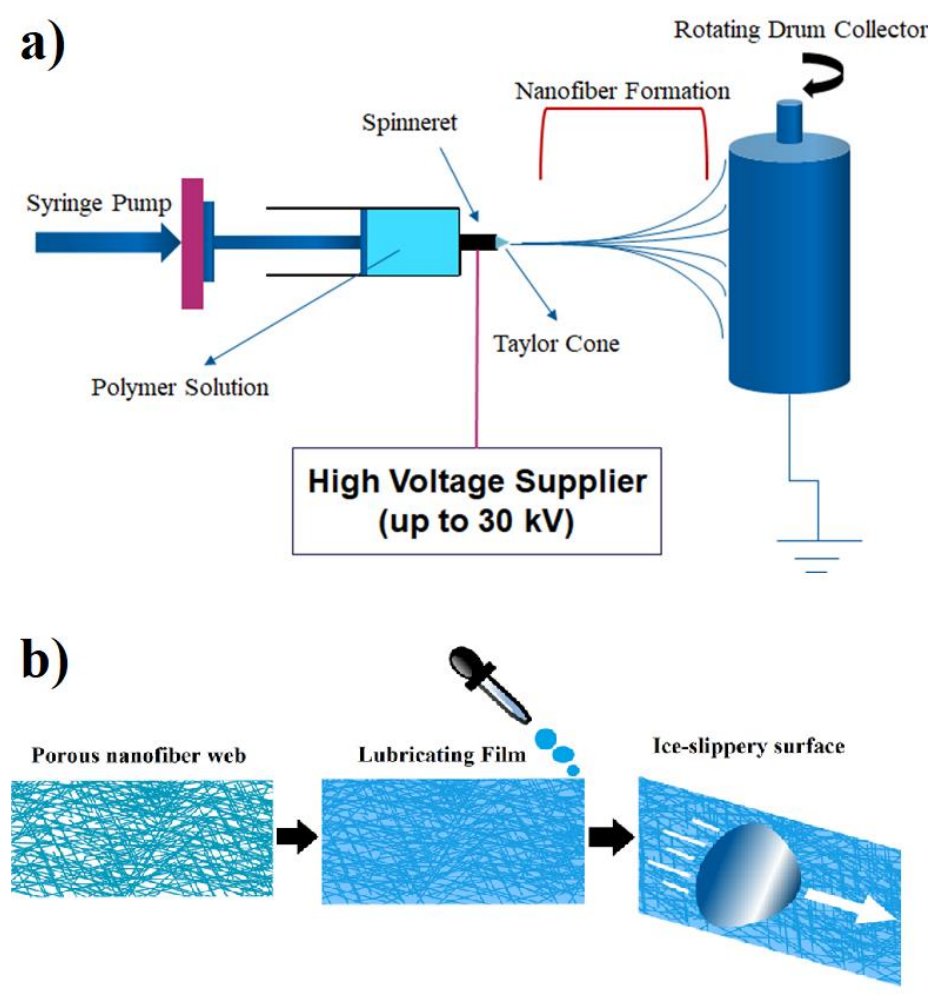

107 Figure 2. (a) Schematic illustration of the electrospinning process and (b) preparation steps of ice-slippery SLIPS

111 Three types of lubricants were used for the fabrication of SLIPS (PcTFE, silicone oil and 112 paraffin wax oil). Approximately, $30 \mathrm{mg} / \mathrm{cm}^{2}$ of lubricant was infused into the porous

113 electrospun nanofibre membrane with a Pasteur pipette upon a balance. All samples were kept 114 in a $45^{\circ}$ tilted plate for overnight to get rid of excess oil and approximately $40 \mu \mathrm{m}$ of thickness 115 was obtained. The preparation steps of the SLIPS are given in Figure 2 (b).

\subsection{Microstructural and performance characterisation}

117 Surface morphology of the electrospun membranes was investigated using scanning electron 118 microscope (Joel 7000), and ImageJ was used to analyse the diameter distribution of fibres 
with 50 measurements. The porosity of the electrospun membrane was investigated by the volume-mass calculation.

For the topographic characterisation, Zeta-20 profilometer, which has ability to analyse transparent surfaces, was used with $50 \mathrm{x}$ magnification. Surface roughness, height profiles and topographic images were obtained with these analyses.

Static and dynamic contact angles of the samples were measured using a FTA200 dynamic contact angle system and the contact angle hysteresis of samples were calculated using Eq. (1).

where $\theta_{\text {hyst }}$ is contact angle hysteresis, $\theta_{a d v}$ is the advancing contact angle and $\theta_{\text {rec }}$ is the receding contact angle.

Ice adhesion tests were performed using a home-made setup, according to centrifuge method $[29,30]$. A glaze ice block with $1.3 \mathrm{~g}$ mass was attached on the sample surface in a $-10^{\circ} \mathrm{C}$ chamber for the measurement. Ice adhesion strengths were calculated using Eq. (2).

$$
F=m r \omega^{2}
$$

where $\mathrm{F}$ is the centrifugal force, $\omega$ is the rotation speed at the detachment of the glaze ice block, $m$ is the mass of the ice in kilograms and $r$ is the length of beam [31].

The water droplet icing tests were performed by observing the water droplets on a cold plate setting at $-10^{\circ} \mathrm{C}$ with a constant volume $(4 \mu \mathrm{L})$ on five spots of samples [31]. The average icing duration was recorded to evaluate the anti-icing performance and droplet images were taken every 10 seconds until the droplet was completely frozen.

Optical properties of the samples were evaluated using a biochrom Libra S22 UV/vis spectrometer for the range of 300 to $900 \mathrm{~nm}$ and optical analyses of SLIPS performed with a microscope slide and subtraction carried out by software to find real optical values. 


\section{RESULTS and DISCUSSION}

145

\subsection{Surface morphology}

Porous fibrous membrane along with high porosity between the fibres $(71.6 \pm 4.1 \%)$ was obtained from the electrospinning process, as shown in Figure 3. Most of the fibres had a diameter range between 100 and $400 \mathrm{~nm}$ which can offer a high surface area. It is important because the high surface area can provide more contact area between lubricant and the fibres. Because the PVDF-co-HFP fibres highly oleophilic, higher contact area can offer better capability of containing the lubricant, which is one of the main concern of SLIPS.

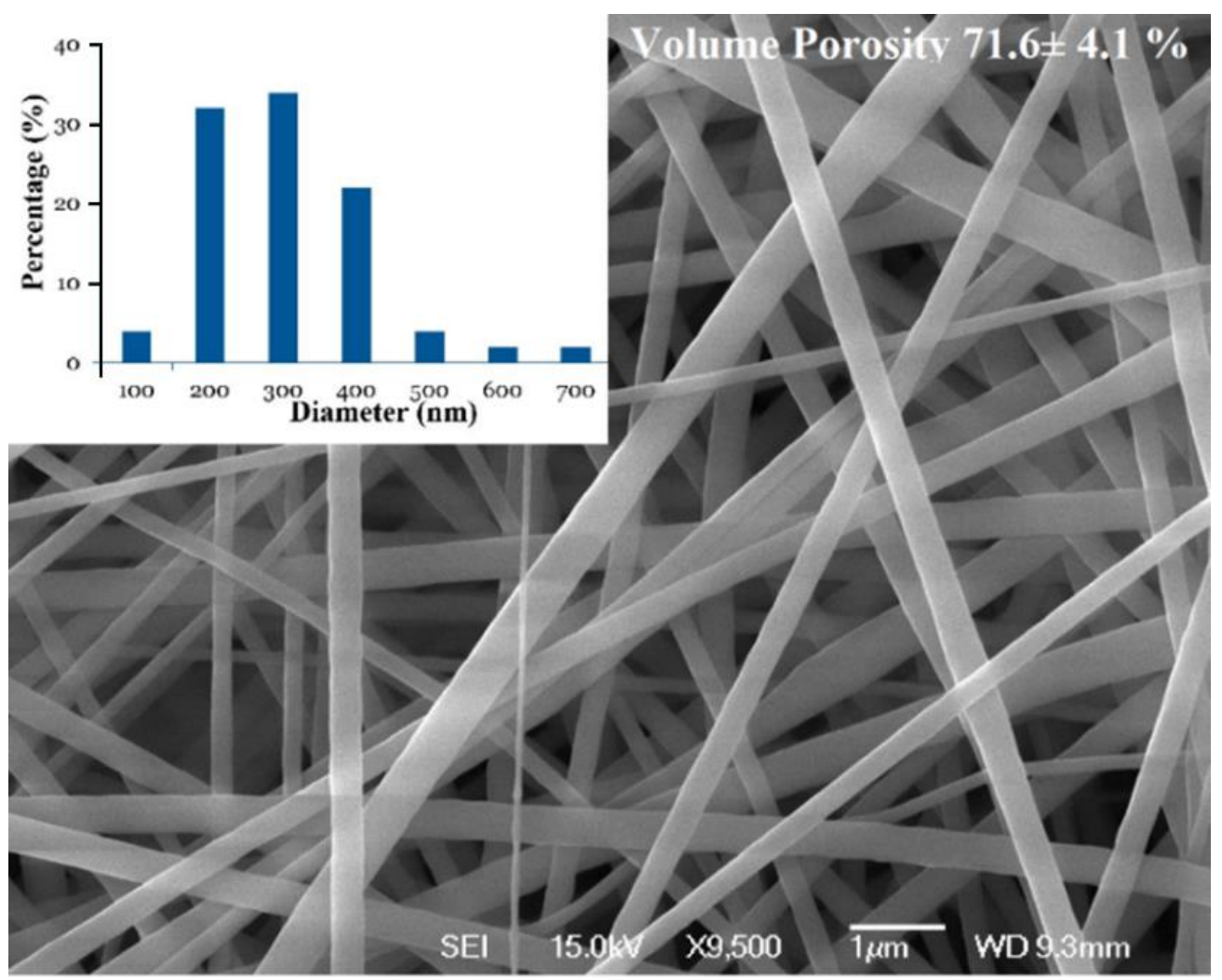

Figure 3. SEM analyses of the as-prepared electrospun membrane (Inset: distribution of fibre diameter)

Surface height profiles of the samples, before and after lubricant infusion, are shown in Figure 4. It was found that the membrane surfaces without lubricant had the maximum peak- 
157 valley heights of approximately $15 \mu \mathrm{m}$. The pores in the structures provided the valleys whilst 158 the overlapping fibres provided the peaks. After the infusion of the lubricants, the 159 maximum peak-valley heights of the samples had decreased to approximately less than $3 \mu \mathrm{m}$ 160 range which is an indication that the lubricant had filled most of the pores. All SLIPS have low 161 peak-valley heights and there is no significant difference between the three lubricants explored, 162 according to height profiles.
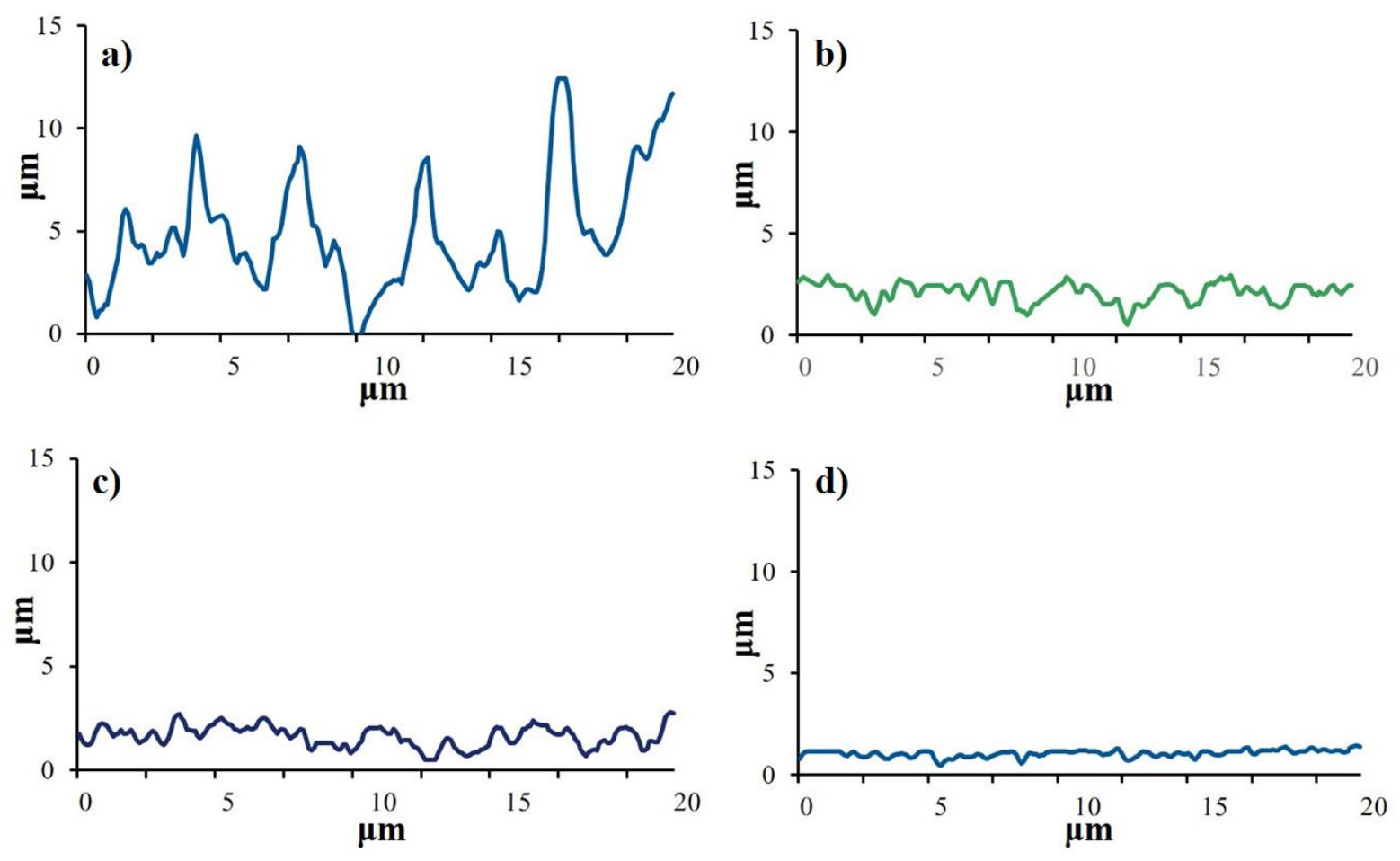

Figure 4. Height profiles of the (a) PVDF-co-HFP electrospun membrane and SLIPS with (b) silicone oil, (c) paraffin oil and (d) PcTFE

The 3D topographic images of the samples are given in Figure 5. The fibrous structure of the electrospun membranes can be reflected in Figure 5 (a) which is a lubricant free structure. After 
the infusion of the lubricant, no fibrous structure could be observed. The roughness of the electrospun membrane also decreased dramatically, consistent with the height profile results.
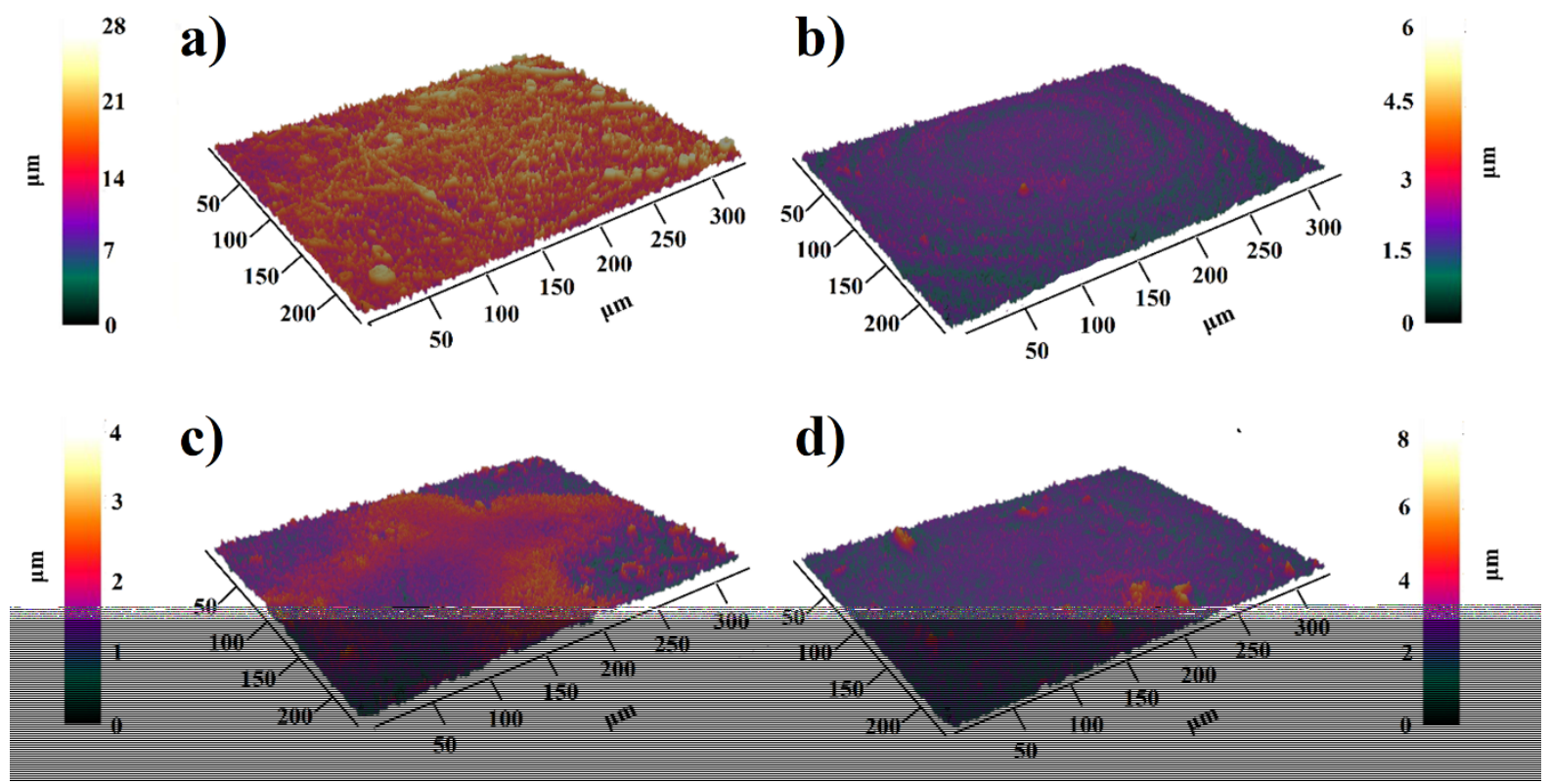

Figure 5. 3D topographic images of (a) PVDF-co-HFP electrospun membrane (b) SLIPS with PcTFE, (c) SLIPS with silicone oil and (d) SLIPS with liquid paraffin

\subsection{Wetting characteristics}

Water contact angles of the SLIPS are shown in Figure 6. The electrospun PVDF-co-HFP membranes produced possessed average contact angle of $141.2^{\circ}$. After the infusion of oils, the roughness and composition of the surface had been changed. So the measured contact angles reflect mostly the nature of the oils used in the SLIPS instead of the fibres. Additionally, according to Cassie-Baxter mechanism, air pockets on the surface have great impact on the hydrophobicity. After the lubricant infusion, theoretically, the air pockets would be significantly reduced with the lubricants which minimised the roughness effect from the asproduced PVDF-co-HFP electrospun membrane structure. Therefore, the static contact angles of the SLIPS decreased dramatically compared to the unfilled fibrous structure, although the 
contact angles were still in the hydrophobic range $\left(>90^{\circ}\right)$. The SLIPS with silicon and paraffin oils have very close contact angles (approximately $98^{\circ}$ ). When the PcTFE was used as lubricant for SLIPS, the surface exhibited a higher contact angle $\left(108^{\circ}\right)$ possibly due to the fluorine containing chemical structures which offered lower surface energy.

190

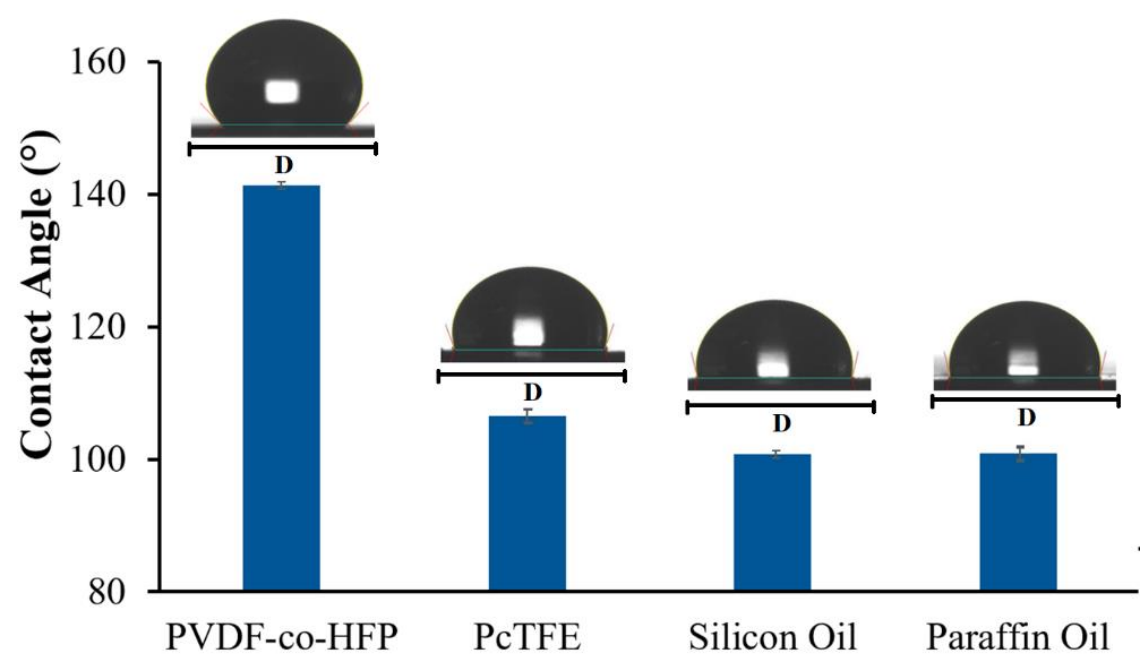

191 Figure 6. Static contact angles results of the as-produced membrane and SLIPS (D=3.0 mm)

192 Contact angle hysteresis of the samples are shown in Figure 7. Although all SLIPS have lower 193 static contact angles, they also have significantly lower contact angle hysteresis than the as194 produced PVDF-co-HFP electrospun membrane. Therefore despite lower contact angle of SLIPS, water droplet would have much higher mobility on the SLIPS compared to lubricant 196 free structure. This is an expected effect of the lubricants reported elsewhere[32]. It is also 197 notable that SLIPS has stable contact angle hysteresis (CAH) with increasing droplet volume. 198 However, the CAH values of the PVDF-co-HFP is significantly dependent on the size of the 199 droplet. The lowest CAH obtained was on the PcTFE SLIPS for the droplet size $3.75 \mu \mathrm{L}$, with approximately $5.5^{\circ}$. 


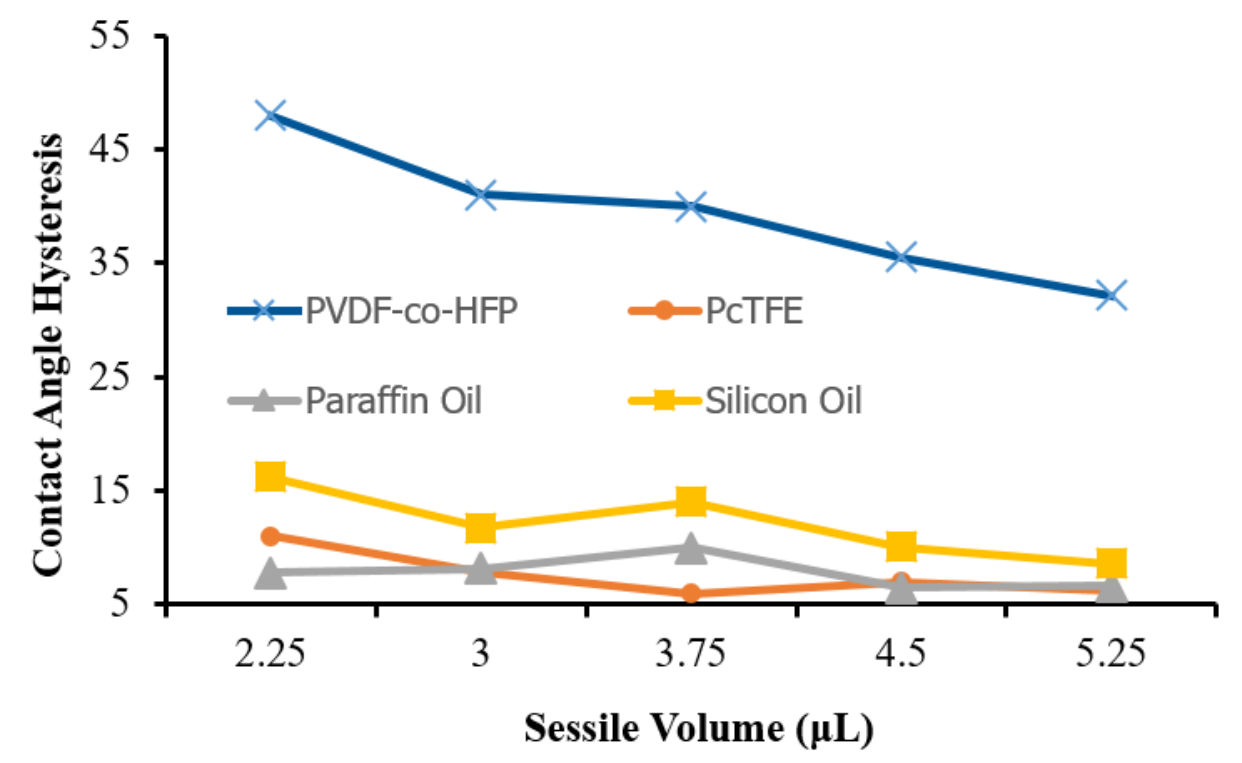

Figure 7. Contact angle hysteresis of the samples

\subsection{Optical properties}

205 Optical transmittances of the samples are shown in Figure 8. PVDF-co-HFP electrospun membrane did not show significant transmittance in the visible light spectrum, between 300 207 and $900 \mathrm{~nm}$ wavelengths. The relationship between deposition time of electrospinning and transparency of PVDF-co-HFP has been discussed before [33] and it was found when the deposition time, or in other words thickness of the membrane increased, the transmittance of the electrospun membrane is decreases. It is well known that transparent materials are made up

211 of components with uniform refractive indices and for multicomponent structures mismatch of 212 the refractive indices results in opacity [34]. In other words, the possibility of light passing 213 through a medium or not, depends on the homogeneity of the refractive index of the final structure. Infusion of the lubricant contributed drastically to the excellent transparency of the

215 electrospun membrane, depending on the refractive index of lubricant used. When silicone oil 216 and PcTFE, which have quite similar refractive indices as PVDF-co-HFP, were used as 217 lubricants, they both provided transmittance higher than $90 \%$ because of the uniform refractive 
index of the entire structure. The lowest transmittance obtained was lower than $40 \%$ when paraffin oil was used as lubricant, which has large discrepancy compared to the refractive index of PVDF-co-HFP. These results are promising because the produced SLIPS may be used for energy harvesting systems where the transparency of the top surface would directly influence 222 the efficiency of the solar panels [35].

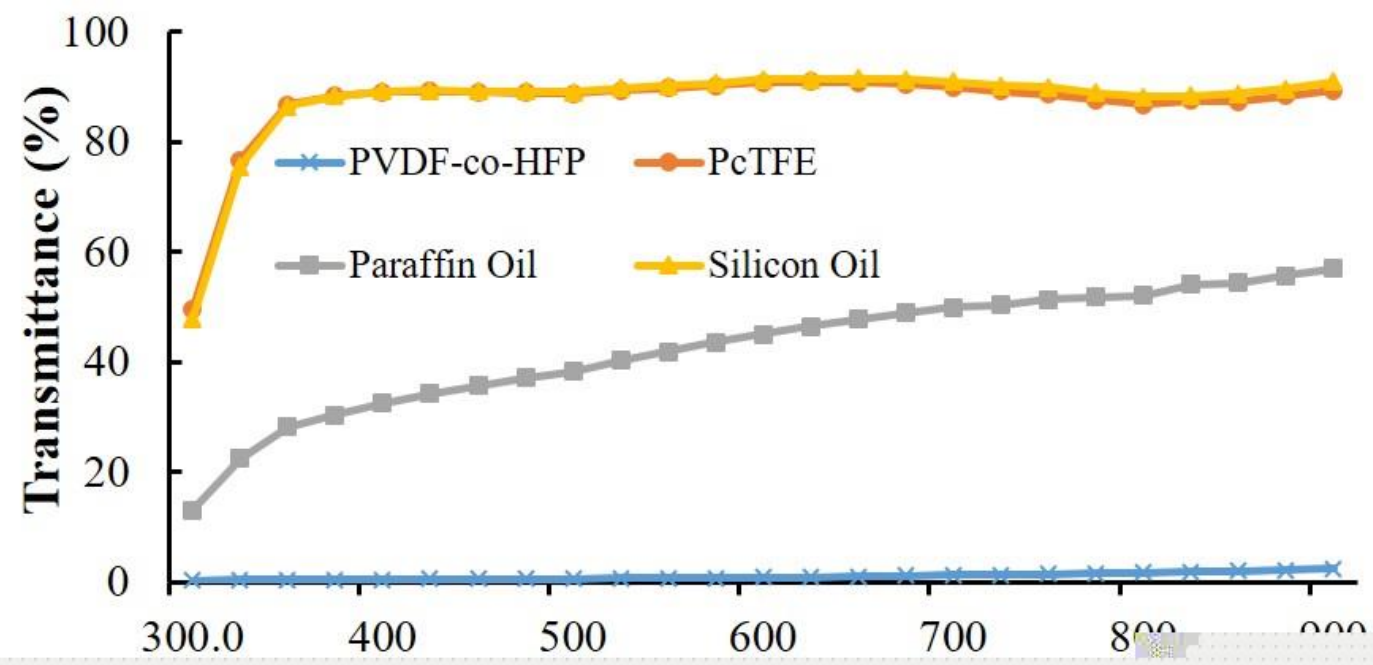

Figure 8. Optical transmittances of samples

\subsection{Ice adhesion and droplet icing time}

227 Ice adhesion test results are presented in Figure 9. The results were compared with the aluminum plate which is one of the most widely used materials for icephobic applications. It was clear that the as-produced PVDF-co-HFP electrospun membrane had much lower ice adhesion strength compared to an aluminum plate. Interestingly, infusion of the lubricant reduced the ice adhesion strength even further. Although all the SLIPS have remarkable values down to less than $1 \mathrm{KPa}$, SLIPS with paraffin oil had the best results with approximately 0.65

$233 \mathrm{KPa}$ ice adhesion strength which is lower than most of the reported studies [36]. The 234 significantly lower ice adhesion strength is associated with the following three aspects: 1) The 
235 immiscibility of water-oil interface prevents water to produce ice anchors into the structure; 2 )

236 SLIPS presents an ultra-smooth surface compared to any other solid and dry surfaces, allowing

237 ice to slide easily; and 3) The SLIPS offers very low surface tension of lubricants compared 238 with the most of solid surfaces. Low surface tension between lubricant-water interfaces 239 effectively prevents the formation of strong adhesion between lubricant and ice. It is also 240 worthy of note that all SLIPS demonstrated much better ice adhesion strength compared to 241 superhydrophobic surfaces which is considered as a good candidate for icephobic applications $242[37]$

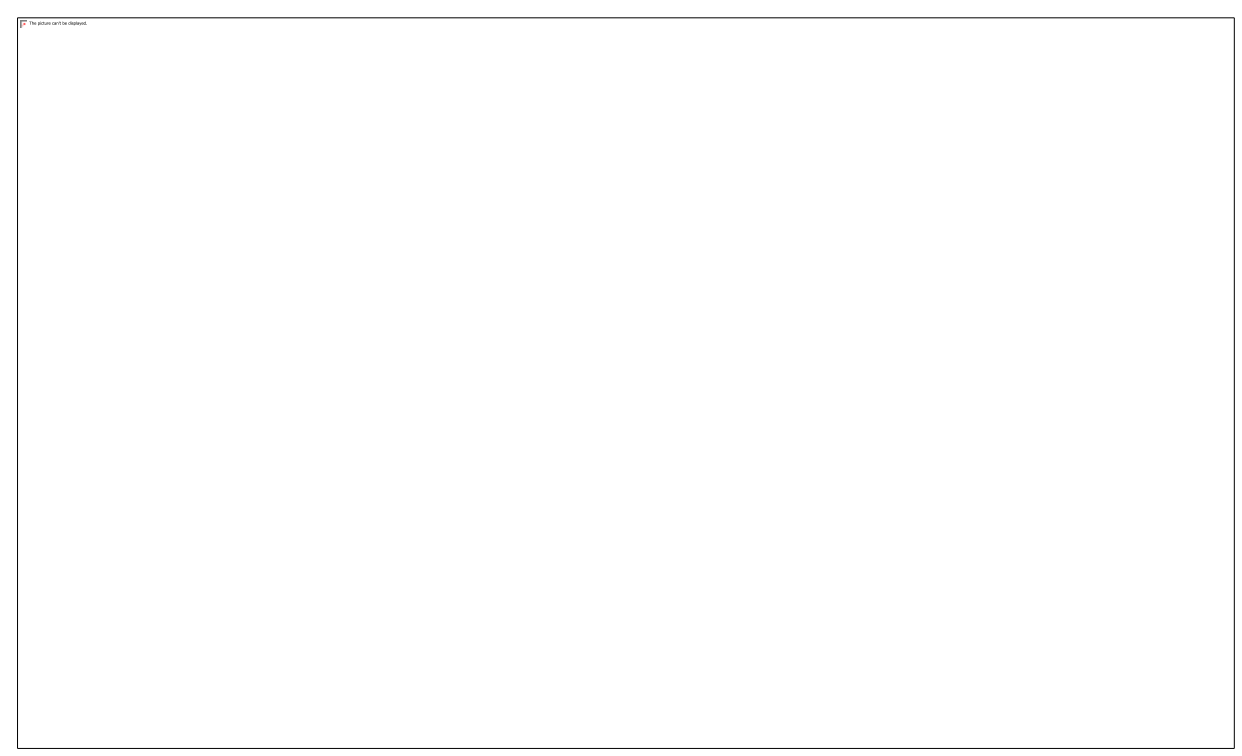

244 Figure 9. Ice adhesion strengths of samples (Inset: ice adhesion test result of SLIPS with silicon oil, paraffin oil and PcTFE, respectively)

246 The results of average droplet icing times with the images during icing process are given in 247 Figure 10. It was found that all of the designed structures had exceptional results compared to 248 the aluminum plate which has 5 seconds freezing time. The PVDF-co-HFP electrospun 249 membrane without any lubricant had the best results with more than 40 seconds icing time. It 250 has already been mentioned that this structure has more than $70 \%$ of porosity that means most 251 of the structure is only 'air` which is a good thermal insulator. This structure also prevented 
water droplets to freeze due to the thermal insulating effect of air. The SLIPS with PcTFE and

253

254

255

256

257

258

259

260

261

262

(a)

263 in icing time. paraffin oil has quite similar anti-icing behaviour with PVDF-co-HFP nanofibre membrane due to their significantly low thermal conductivities. It is believed that the anti-icing properties are more closely related to the surface structure, instead of chemical compositions [38]. However, SLIPS with silicone oil showed much lower icing time because of its much higher heat conduction ability. The thermal conductivities of PcTFE, liquid paraffin and silicone oil are $0.065,0.12$ and $0.6 \mathrm{~W} / \mathrm{m} / \mathrm{K}$, respectively. The water contact angle would also affect the droplet icing time. Lower contact angle of the droplet means larger contact area, then the heat resistance between the tested surface and the droplet would be reduced, causing to a decrease

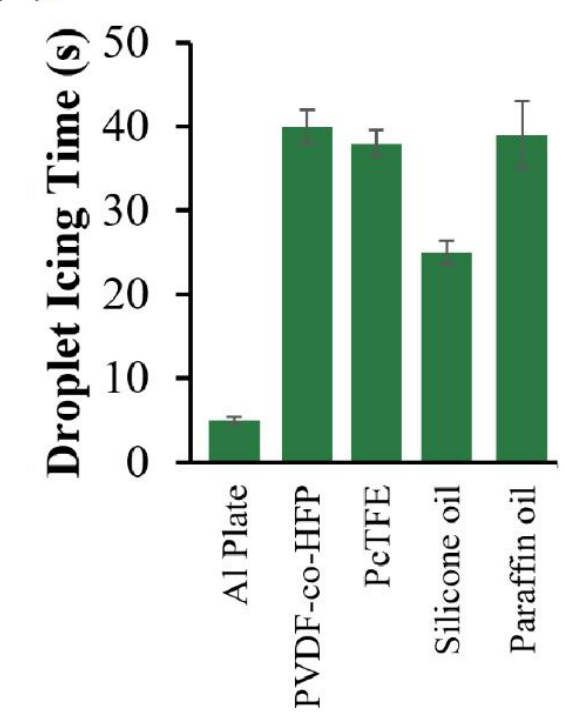

(b)

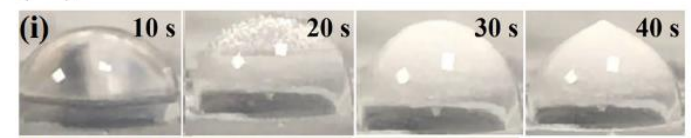

(ii) $\quad 10 \mathrm{~s} \quad 20 \mathrm{~s}$
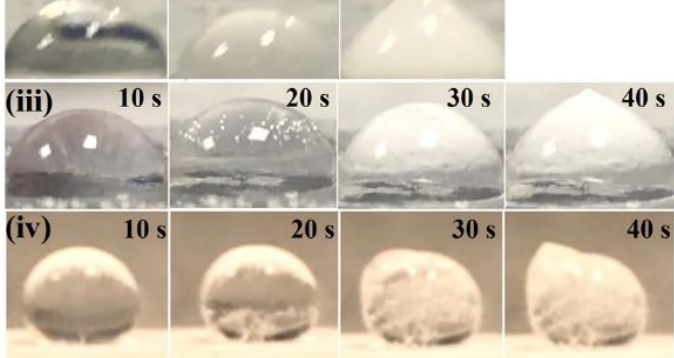

(v) $\quad 10 \mathrm{~s}$

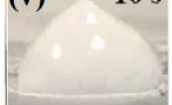




\section{CONCLUSIONS}

269 In this study, we present a cost-effective and scalable electrospinning technique to produce 270 freestanding nanofibrous polymeric surfaces for the fabrication of transparent icephobic 271 SLIPS. Three liquids (PcTFE, silicon oil and paraffin liquid) were used as lubricants. The topographical images of the samples showed that infusion of the lubricant provided smooth surface which is one of the critical parameters for icephobic application. It was also found that the mobility of the droplets on the SLIPS was enhanced dramatically compared to the asproduced PVDF-co-HFP electrospun membrane. Because of the quite similar refractive indices of lubricants and polymer, SLIPS with silicone oil and PcTFE showed transmittance as high as $90 \%$ in the visible light spectrum. All SLIPS exhibited low ice adhesion strength down to 1 $\mathrm{KPa}$ with icing delay time from 5 to 41 seconds. It is promising that these SLIPS surface can be used for transparency and icephobicity required applications.

\section{Acknowledgment}

281

282

The authors would like to thank the Scientific and Technological Research Council of Turkey (TUBITAK) for providing financial support to MAHMUT TAS with the program number 2213, the Nanoscale and Microscale Research Centre (nmRC) at University of Nottingham for the access to instrumentation and Halocarbon for offering free PcTFE oil.

\section{References}

[1] R. Menini, M. Farzaneh, Advanced Icephobic Coatings, J Adhes Sci Technol, 25 (2011) 971-992.

[2] Y.Z. Zhuo, F. Wang, S.B. Xiao, J.Y. He, Z.L. Zhang, One-Step Fabrication of Bioinspired Lubricant-Regenerable Icephobic Slippery Liquid-Infused Porous Surfaces, Acs Omega, 3 (2018) 10139-10144.

[3] H.K. Zheng, S.N. Chang, Y.Y. Zhao, Anti-Icing \& Icephobic Mechanism and Applications of Superhydrophobic/Ultra Slippery Surface, Prog Chem, 29 (2017) 102-118.

[4] P. Irajizad, M. Hasnain, N. Farokhnia, S.M. Sajadi, H. Ghasemi, Magnetic slippery extreme icephobic surfaces, Nat Commun, 7 (2016) 13395. 
[5] O. Parent, A. Ilinca, Anti-icing and de-icing techniques for wind turbines: Critical review, Cold Reg Sci Technol, 65 (2011) 88-96. [6] C. Murphy, S. Wallace, R. Knight, D. Cooper, T. Sellers, Treatment performance of an aerated constructed wetland treating glycol from de-icing operations at a UK airport, Ecol Eng, 80 (2015) 117-124.

[7] C.R.d.A. de Andrés, S. Saarinen, A. Uuskallio, REVIEW OF ICE CHALLENGES AND ICE MANAGEMENT IN PORT AREAS, Coastal Engineering Proceedings, 1 (2018) 79.

[8] T.S. Wong, S.H. Kang, S.K.Y. Tang, E.J. Smythe, B.D. Hatton, A. Grinthal, J. Aizenberg, Bioinspired self-repairing slippery surfaces with pressure-stable omniphobicity, Nature, 477 (2011) 443-447.

[9] S.B. Subramanyam, K. Rykaczewski, K.K. Varanasi, Ice Adhesion on LubricantImpregnated Textured Surfaces, Langmuir, 29 (2013) 13414-13418.

[10] R.M. Dou, J. Chen, Y.F. Zhang, X.P. Wang, D.P. Cui, Y.L. Song, L. Jiang, J.J. Wang, Anti-icing Coating with an Aqueous Lubricating Layer, Acs Appl Mater Inter, 6 (2014) 69987003.

[11] S. Ozbay, C. Yuceel, H.Y. Erbil, Improved Icephobic Properties on Surfaces with a Hydrophilic Lubricating Liquid, Acs Appl Mater Inter, 7 (2015) 22067-22077.

[12], N. Wang, L. Tang, Y. Cai, D. Xiong,. Lyophobic slippery surfaces on smooth/hierarchical structured substrates and investigations of their dynamic liquid repellency, Phys Chem Chem Phys, 21, (2019) 15705-15711.

[13] L. Zhu, J. Xue, Y.Y. Wang, Q.M. Chen, J.F. Ding, Q.J. Wang, Ice-phobic Coatings Based on Silicon-Oil-Infused Polydimethylsiloxane, Acs Appl Mater Inter, 5 (2013) 40534062.

[14] C.Q. Wei, B.Y. Jin, Q.H. Zhang, X.L. Zhan, F.Q. Chen, Anti-icing performance of superwetting surfaces from icing-resistance to ice-phobic aspects: Robust hydrophobic or slippery surfaces, J Alloy Compd, 765 (2018) 721-730.

[15] X.H. Wu, S.L. Zheng, D.A. Bellido-Aguilar, V.V. Silberschmidt, Z. Chen, Transparent icephobic coatings using bio-based epoxy resin, Mater Design, 140 (2018) 516-523.

[16] X.H. Wu, Z. Chen, A mechanically robust transparent coating for anti-icing and selfcleaning applications, J Mater Chem A, 6 (2018) 16043-16052.

[17] L.Q. Chen, A. Geissler, E. Bonaccurso, K. Zhang, Transparent Slippery Surfaces Made with Sustainable Porous Cellulose Lauroyl Ester Films, Acs Appl Mater Inter, 6 (2014) 6969-6976.

[18] F.J. Wang, S. Yu, J.F. Ou, W. Li, Anti-icing performance of transparent and superhydrophobic surface under wind action, J Sol-Gel Sci Techn, 75 (2015) 625-634.

[19] Y.Z. Shen, X.Y. Xie, Y.H. Xie, J. Tao, J.W. Jiang, H.F. Chen, Y. Lu, Y.J.S. Xu, Statistically understanding the roles of nanostructure features in interfacial ice nucleation for enhancing icing delay performance, Phys Chem Chem Phys, 21 (2019) 19785-19794. [20] P. Wang, Z. Lu, D. Zhang, Slippery liquid-infused porous surfaces fabricated on aluminum as a barrier to corrosion induced by sulfate reducing bacteria, Corros Sci, 93 (2015) 159-166.

[21] X. Zhou, Y.Y. Lee, K.S.L. Chong, C.B. He, Superhydrophobic and slippery liquidinfused porous surfaces formed by the self-assembly of a hybrid $A B C$ triblock copolymer and their antifouling performance, J Mater Chem B, 6 (2018) 440-448.

[22] J.L. Yong, F. Chen, Q. Yang, Y. Fang, J.L. Huo, J.Z. Zhang, X. Hou, Nepenthes Inspired Design of Self-Repairing Omniphobic Slippery Liquid Infused Porous Surface (SLIPS) by Femtosecond Laser Direct Writing, Adv Mater Interfaces, 4 (2017) 1700552.

[23] J.L. Yong, J.L. Huo, Q. Yang, F. Chen, Y. Fang, X.J. Wu, L. Liu, X.Y. Lu, J.Z. Zhang, $X$. Hou, Femtosecond Laser Direct Writing of Porous Network Microstructures for Fabricating Super-Slippery Surfaces with Excellent Liquid Repellence and Anti-Cell Proliferation, Adv Mater Interfaces, 5 (2018) 1701479.

[24] I. Okada, S. Shiratori, High-Transparency, Self-Standable Gel-SLIPS Fabricated by a Facile Nanoscale Phase Separation, Acs Appl Mater Inter, 6 (2014) 1502-1508.

[25] Q. Liu, Y. Yang, M. Huang, Y.X. Zhou, Y.Y. Liu, X.D. Liang, Durability of a lubricantinfused Electrospray Silicon Rubber surface as an anti-icing coating, Appl Surf Sci, 346 (2015) 68-76. 
[26] T. Dumont, T. Lippert, A. Wokaun, P. Leyvraz, Laser writing of 2D data matrices in glass, Thin Solid Films, 453 (2004) 42-45.

[27] W. Lu, A.M. Sastry, Self-assembly for semiconductor industry, Ieee T Semiconduct M, 20 (2007) 421-431.

[28] S.K. Nune, K.S. Rama, V.R. Dirisala, M.Y. Chavali, Chapter 11 - Electrospinning of collagen nanofiber scaffolds for tissue repair and regeneration, in: D. Ficai, A.M. Grumezescu (Eds.) Nanostructures for Novel Therapy, Elsevier2017, pp. 281-311.

[29] C. Laforte, A. Beisswenger, Icephobic material centrifuge adhesion test, Proceedings of the 11th International Workshop on Atmospheric Icing of Structures, IWAIS, Montreal, QC, Canada, 2005, pp. 12-16.

[30] G. Fortin, A. Beisswenger, J. Perron, Centrifuge adhesion test to evaluate icephobic coatings, AIAA Atmospheric and Space Environments Conference, 2010, pp. 7837.

[31] J.P. Liu, Z.A. Janjua, M. Roe, F. Xu, B. Turnbull, K.S. Choi, X.H. Hou, SuperHydrophobic/Icephobic Coatings Based on Silica Nanoparticles Modified by Self-Assembled Monolayers, Nanomaterials, 6 (2016) 232.

[32] J.D. Smith, R. Dhiman, S. Anand, E. Reza-Garduno, R.E. Cohen, G.H. McKinley, K.K. Varanasi, Droplet mobility on lubricant-impregnated surfaces, Soft Matter, 9 (2013) 17721780.

[33] J. Abe, M. Tenjimbayashi, S. Shiratori, Electrospun nanofiber SLIPS exhibiting high total transparency and scattering, Rsc Adv, 6 (2016) 38018-38023.

[34] P. Tao, Y. Li, A. Rungta, A. Viswanath, J.N. Gao, B.C. Benicewicz, R.W. Siegel, L.S. Schadler, $\mathrm{TiO} 2$ nanocomposites with high refractive index and transparency, J Mater Chem, 21 (2011) 18623-18629.

[35] R.M. Fillion, A.R. Riahi, A. Edrisy, A review of icing prevention in photovoltaic devices by surface engineering, Renew Sust Energ Rev, 32 (2014) 797-809.

[36] Y. Shen, X. Wu, J. Tao, C. Zhu, Y. Lai, Z. Chen, Icephobic materials: fundamentals, performance evaluation, and applications, Progress in Materials Science, 103 (2019) 509557.

[37] M.J. Kreder, J. Alvarenga, P. Kim, J. Aizenberg, Design of anti-icing surfaces: smooth, textured or slippery?, Nat Rev Mater, 1 (2016) 15003.

[38] N. Wang, L.L. Tang, W. Tong, D.S. Xiong, Fabrication of robust and scalable superhydrophobic surfaces and investigation of their anti-icing properties, Mater Design, 156 (2018) 320-328. 